\title{
Lumière et art, ou comment (perce)voir l'invisible
}

Lucile Beck (lucile.beck@cea.fr)

Laboratoire de Mesure du Carbone 14 (CEA/CNRS/IRD/IRSN/MCC), CEA Saclay, 91191 Gif-sur-Yvette Cedex

Lorsqu'un tableau,

une sculpture ou un objet

est envoyé au laboratoire

pour étude, il suit une série

d'examens comme...

un patient arrivant à l'hôpital.

Pour l'examen des peintures en particulier, les différentes lumières - rayons $\mathrm{X}$, ultraviolets, visibles, infrarouges caressent les surfaces picturales ou, au contraire, explorent les couches sous-jacentes grâce à des rayonnements pénétrants. On peut ainsi « voir l'invisible» et découvrir ou redécouvrir des dessins préparatoires, des signatures cachées ou des œuvres perdues.

L'objet de cet article est de présenter les différentes techniques et de les illustrer par des investigations récentes.

\section{Les différentes lumières pour l'examen des peintures}

Une peinture est un mille-feuilles constitué de matériaux variés qui, allant du support à la surface extérieure comprend, de façon schématique, les couches suivantes : un panneau de bois ou une toile tendue sur un cadre, une ou deux couches de préparation visant à égaliser la surface, une esquisse ou un dessin préparatoire (optionnel), des couches de peinture (de une à plus de dix selon les œuvres), une ou plusieurs couches de glacis (optionnelles) et enfin un vernis ${ }^{(a)}$. Notre œil ne perçoit que les dernières couches, et parfois peu d'indices laissent deviner les compositions sous-jacentes.

Pour mieux comprendre les réalisations, il faut l'aide de rayonnements (i) plus pénétrants, comme les rayons $\mathrm{X}$ ou infrarouges, pour sonder pas à pas les différentes strates, ou (ii) plus sélectifs pour détecter des composés particuliers.
La série d'examens qui va être décrite dans cet article est basée sur le savoirfaire et l'expérience des conservateurs, ingénieurs et photographes du Centre de recherche et de restauration des musées de France (C2RMF), laboratoire établi au Louvre depuis 1931. Le protocole qu'ils ont bâti pour les peintures est aussi appliqué, à quelques nuances près, aux objets et sculptures.

Pour illustrer ces techniques, nous nous appuierons principalement sur trois tableaux conservés au musée du Louvre et présentés dans la figure 1 : deux œuvres célèbres de Léonard de Vinci (1452-1519) : La Joconde (1503-1506) [1] et Saint Jean-Baptiste (1513-1516), ainsi qu'un tableau anonyme du XVI ${ }^{\mathrm{e}}$ siècle, Dame priant.

Après des observations visuelles, à l'œil nu ou sous loupe, les œuvres sont explorées à l'aide de rayonnements visibles et invisibles (ultraviolets, infrarouges et rayons $\mathrm{X})$.

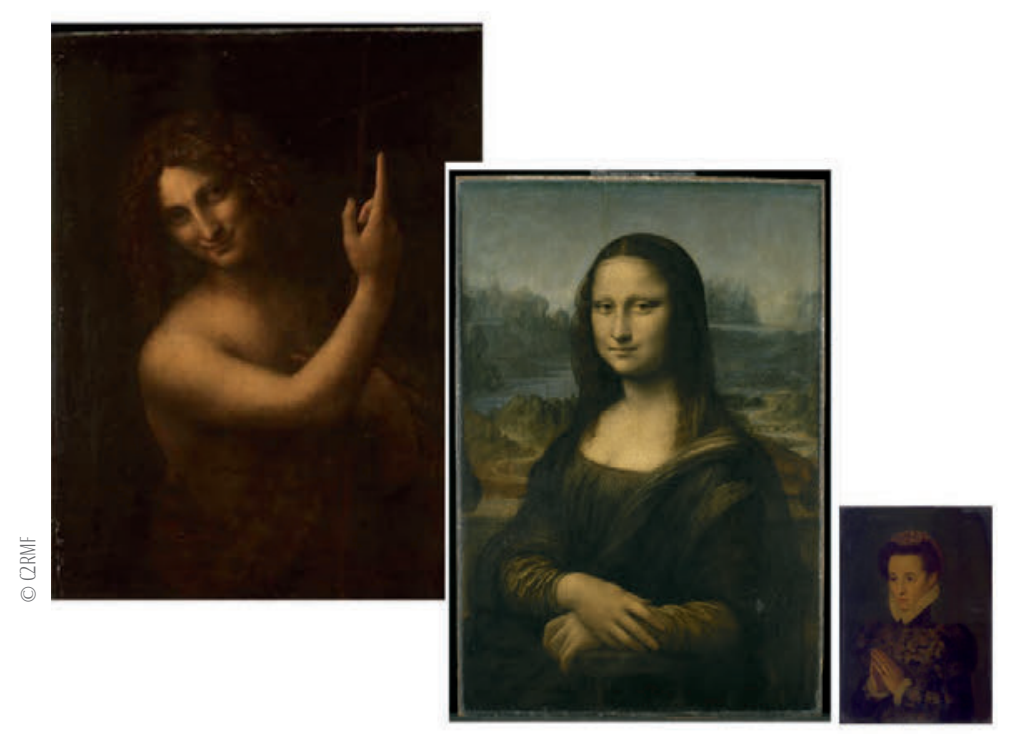

Remerciements

L'auteur remercie Elsa Lambert, photographe et radiologue au C2RMF, pour les précision apportées sur l'instrumentation.

1. Photographie en lumière visible des trois tableaux présentés (mis à l'échelle l'un par rapport à l'autre). De gauche à droite : Saint Jean-Baptiste, de Léonard de Vinci ; La Joconde, de Léonard de Vinci ; Dame priant, œuvre anonyme du XVIe siècle. Musée du Louvre. 


\section{Photographie en lumière visible}

Des photographies en couleurs, et parfois en noir et blanc, sont effectuées sur le verso et le recto de la peinture. Elles permettent de visualiser l'ensemble de l'œuvre (fig. 1) et, grâce à la haute définition, elles donnent accès aux très petits détails $^{(b)}$. Ceux-ci peuvent concerner la réalisation de la peinture elle-même ou son état de conservation. La même lumière, dirigée de façon rasante, permet d'obtenir des photographies qui mettent en valeur les reliefs de l'œuvre en utilisant le rendu visuel des ombres portées. On peut discerner, par exemple, pour la construction du support les marques de couture des toiles ou les traverses des panneaux de bois ; des fentes et autres accidents touchant celui-ci ou la couche picturale peuvent être mis en évidence. Les détails de réalisation, comme les coups de pinceaux ou de couteaux sont aussi exacerbés.

Pour poursuivre l'exploration de l'œuvre, c'est-à-dire " voir " sous la surface ou mettre en évidence des contrastes de compositions, des examens non destructifs à l'aide de rayonnements ultraviolets, infrarouges et $\mathrm{X}$ sont pratiqués.

\section{Photographie en fluorescence de rayons ultraviolets}

Le principe consiste à étudier la propriété de certains matériaux à émettre une fluorescence visible sous éclairage ultraviolet (UV). Ce phénomène touche plus particulièrement les vernis composés de molécules organiques. Ils émettent une lumière bleutée-verte sous rayonnement UV, à la différence des peintures qui y sont généralement peu sensibles ${ }^{(c)}$.

Les clichés ainsi réalisés permettent de détecter rapidement, par contraste de luminosité, des restaurations postérieures à la pose d'un vernis. Consistant en retouches de peinture, celles-ci apparaissent

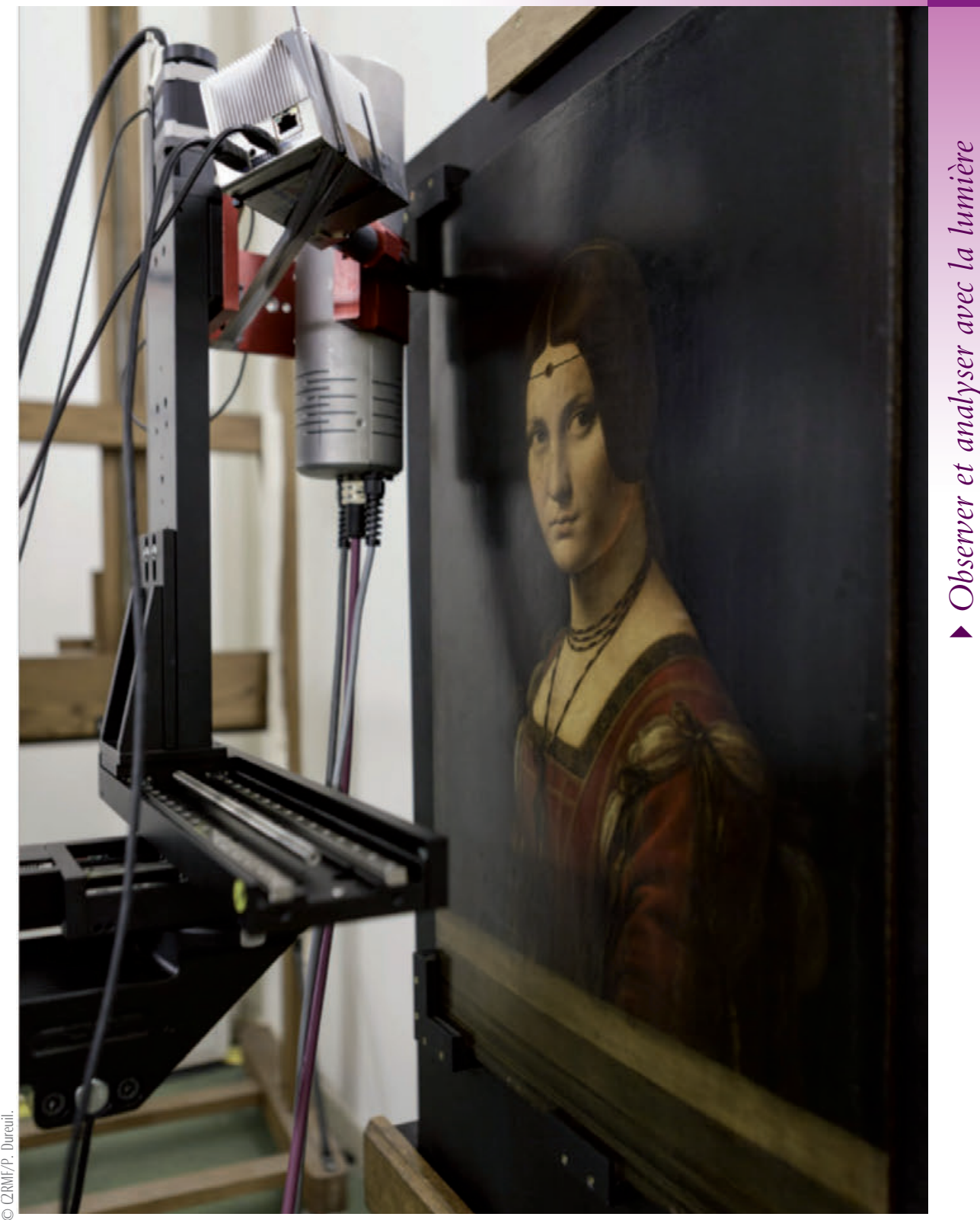

Analyse par fluorescence X de La Belle Ferronnière de Léonard de Vinci [10]. Voir texte, p. 104.

plus sombres que la couche protectrice fluorescente.

Lors de la prise de vue, le sujet ou la peinture sont éclairés avec des lampes UV émettant à une longueur d'onde $\lambda=365 \mathrm{~nm}$. Devant l'objectif de l'appareil photographique, il est alors nécessaire de placer un filtre éliminant les radiations de longueur d'onde inférieure à $420 \mathrm{~nm}$ (lumière UV diffusée ou réfléchie) et ne laissant passer que la lumière visible correspondant à la fluorescence de l'objet.

La figure 2 (p. 102) illustre trois cas très différents d'état de surface de tableaux éclairés en lumière UV. On reconnaît, à l'intensité et l'uniformité de la fluorescence du tableau représentant Saint Jean-Baptiste, un vernis homogène et épais. En revanche, de nombreuses hétérogénéités sont observées pour les deux autres peintures. Ces hétérogénéités sont dues, d'une part, à l'épaisseur irrégulière du vernis et, d'autre part, à la présence de restaurations qui ont été apposées sur le vernis plus ancien.

On peut distinguer en bas, à droite du tableau La Joconde une restauration moderne ; elle est consécutive aux dommages causés, le 30 décembre 1956, par un visiteur du Louvre qui avait lancé un projectile sur l'œuvre exposée. Sur le haut du tableau, ce sont des « repeints » (d) plus anciens qui sont mis en évidence ; ceux-ci correspondent à une fissure du panneau de bois, qui a entraîné une altération de la couche picturale [1].

Sur le tableau Dame priant, des retouches postérieures encore plus nombreuses sont visibles, témoignant de multiples restaurations. 
$>>$

La photographie ou réflectographie infrarouge

Les rayonnements infrarouges (IR) ont la capacité de traverser les couches picturales pour atteindre les tracés sous-jacents. En effet, dans ce domaine de longueurs d'onde (1 à $2 \mu \mathrm{m})$, la plupart des pigments sont transparents alors que les matériaux à base d'un composé noir - carbone pour le fusain ou oxyde de manganèse - sont fortement absorbants [2]. Les dessins sous-jacents de peintures anciennes peuvent être ainsi révélés, dès lors que les esquisses ont été exécutées en noir sur fond blanc. Pour les peintures plus récentes, où les compositions ont été plus souvent réalisées à l'encre ou à la craie, le contraste est plus faible et les tracés moins visibles. Enfin, la visualisation des tracés sous-jacents est difficile dans le cas de couches de peinture composées ellesmêmes de pigments noirs, puisqu'elles absorbent aussi les IR.

La photographie infrarouge opère avec des éclairages identiques à ceux utilisés pour les prises de vues en lumière directe. Les radiations IR sont enregistrées avec des capteurs CCD sensibles de 400 à $1000 \mathrm{~nm}$. Un filtre est placé devant l'objectif pour éliminer la lumière visible.
La réflectographie IR possède un système de détection équipé d'un détecteur semiconducteur (InGa)As. Plus sensible aux grandes longueurs d'onde (> $1000 \mathrm{~nm})$, il permet de traverser des matériaux plus opaques ou d'explorer des couches plus profondes. De plus, l'utilisation d'un filtre n'est plus nécessaire.

La mise en œuvre des techniques IR peut mettre au jour des signatures cachées, ou révéler les hésitations ou " repentirs » $(\mathrm{d})$ des artistes. Ainsi, on peut voir sur les détails de la figure 3 que L. de Vinci a modifié la position des doigts des personnages, entre le début et la fin de l'exécution des tableaux. Toujours sur une ouvre de L. de Vinci, La Vierge, l'Enfant Jésus et Sainte Anne, mais cette fois-ci au verso, ce qui est peu courant, la réflectographie IR a dévoilé la présence de dessins très peu visibles à l'œil nu (fig. 4a). C'est au moment de l'examen du tableau en prévision de sa restauration, que des traits à peine perceptibles ont attiré l'œil d'un conservateur du département des peintures du musée du Louvre. Difficilement reconnaissables, ils ont été révélés lors des prises de vue sous IR (fig. 4b). Ainsi, des dessins préparatoires représentant un enfant Jésus à l'agneau, un crâne et une tête de cheval ont été mis au jour. La confrontation de ces dessins avec d'autres œuvres du peintre a permis de lui en attribuer la paternité. Cette découverte est exceptionnelle, car les dessins exécutés au revers d'œuvres sont très rares et même inédits dans le cas de Léonard de Vinci [3].

\section{La radiographie}

La radiographie $\mathrm{X}$ est la technique scientifique la plus ancienne pour l'investigation des peintures. Elle a été utilisée dès l'année qui a suivi la découverte des rayons $\mathrm{X}$, à la fin du $\mathrm{XIX}^{\mathrm{e}}$ siècle. Elle s'est développée ensuite en Allemagne, puis aux Pays-Bas. En France, la première expérience de radiographie de tableaux a été rapportée à l'Académie des sciences, le 3 janvier 1921. Contrairement aux techniques photographiques qui enregistrent la lumière réfléchie, la radiographie $\mathrm{X}$ mesure les rayonnements transmis à travers l'objet. Elle permet ainsi l'exploration de l'intérieur de l'œuvre, afin d'établir un diagnostic sur son état de conservation ou de déterminer les différents assemblages qui la composent. Pour les peintures, on obtient des
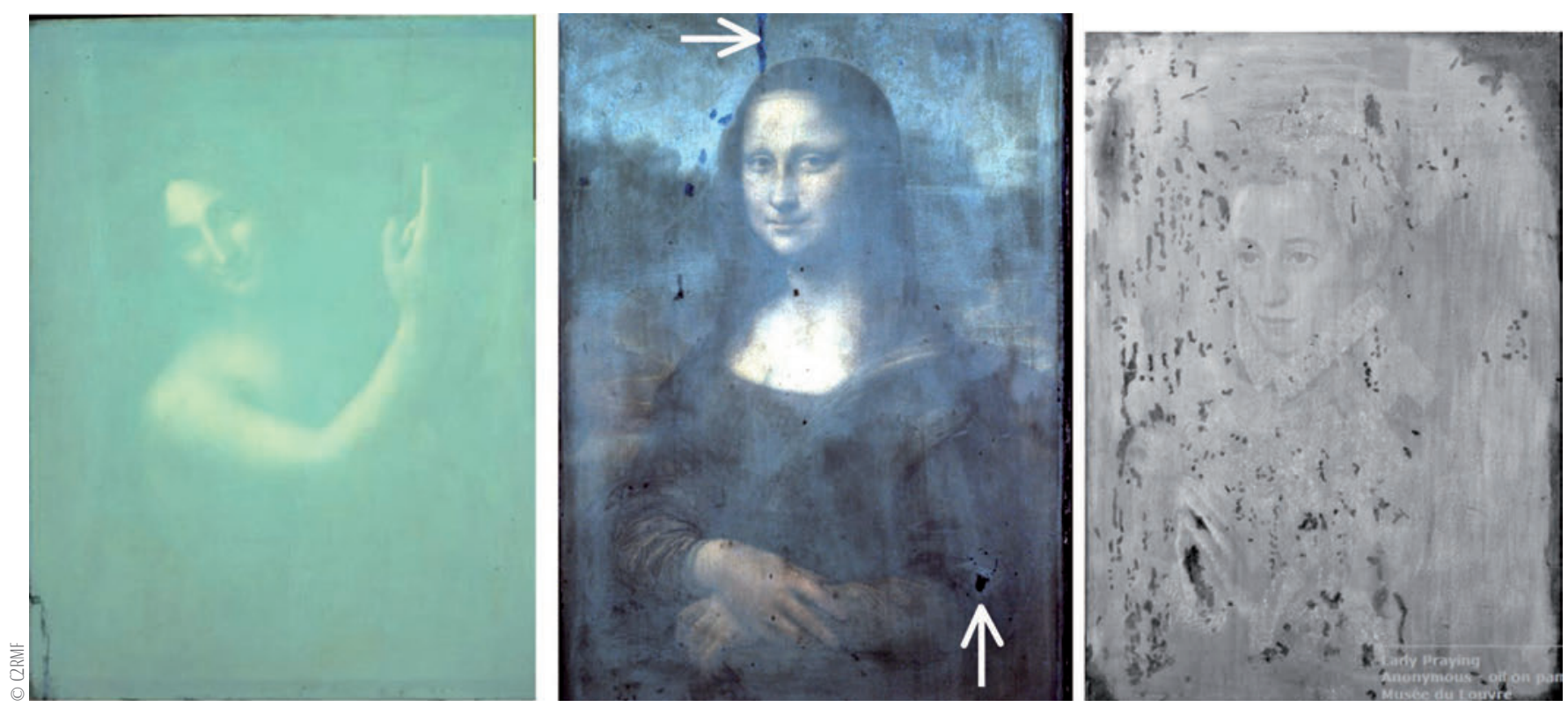

2. Photographie sous éclairage ultraviolet $(\lambda=365 \mathrm{~nm})$ des trois tableaux présentés en figure 1 . Les restaurations effectuées sur le vernis apparaissent sous forme de taches sombres contrastant avec la fluorescence " laiteuse » du vernis. Deux d'entre elles sont indiquées par des flèches sur le tableau de La Joconde. 


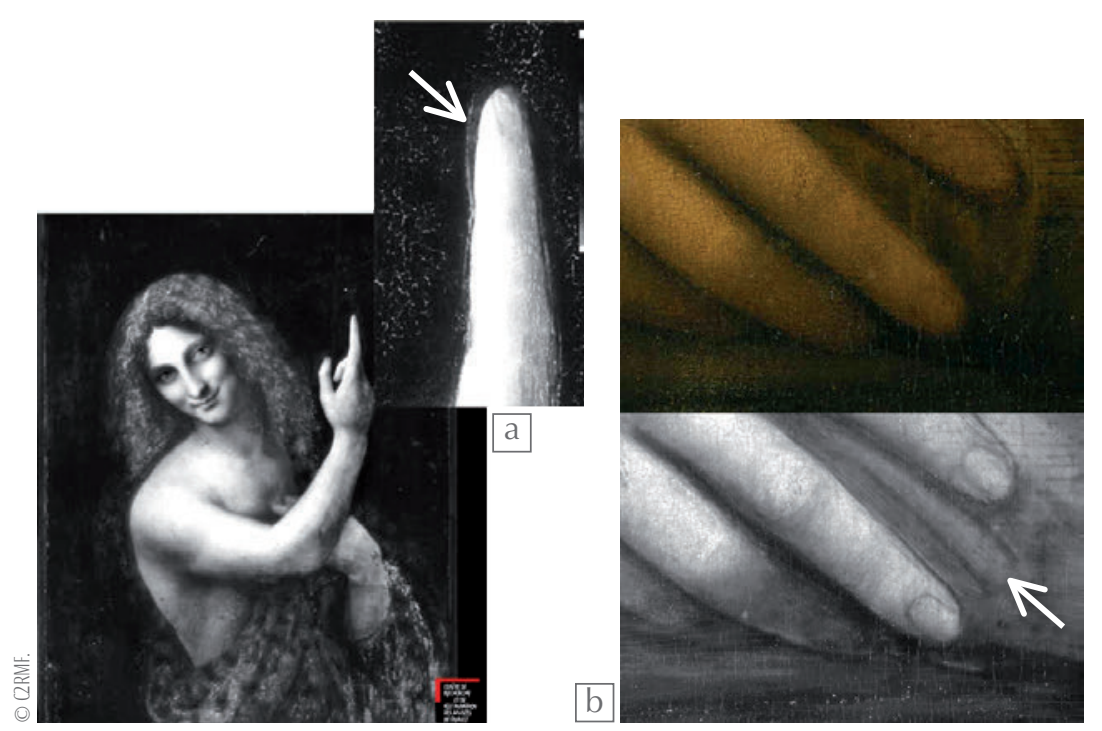

3. Photographies en réflectométrie infrarouge mettant en évidence de légers repentirs dans l'emplacement des doigts. (a) Saint Jean-Baptiste, avec détail sur l'index. (b) Détail de la main droite de La Joconde. La même partie en photographie visible (en haut) permet de le comparer avec l'œuvre finale.

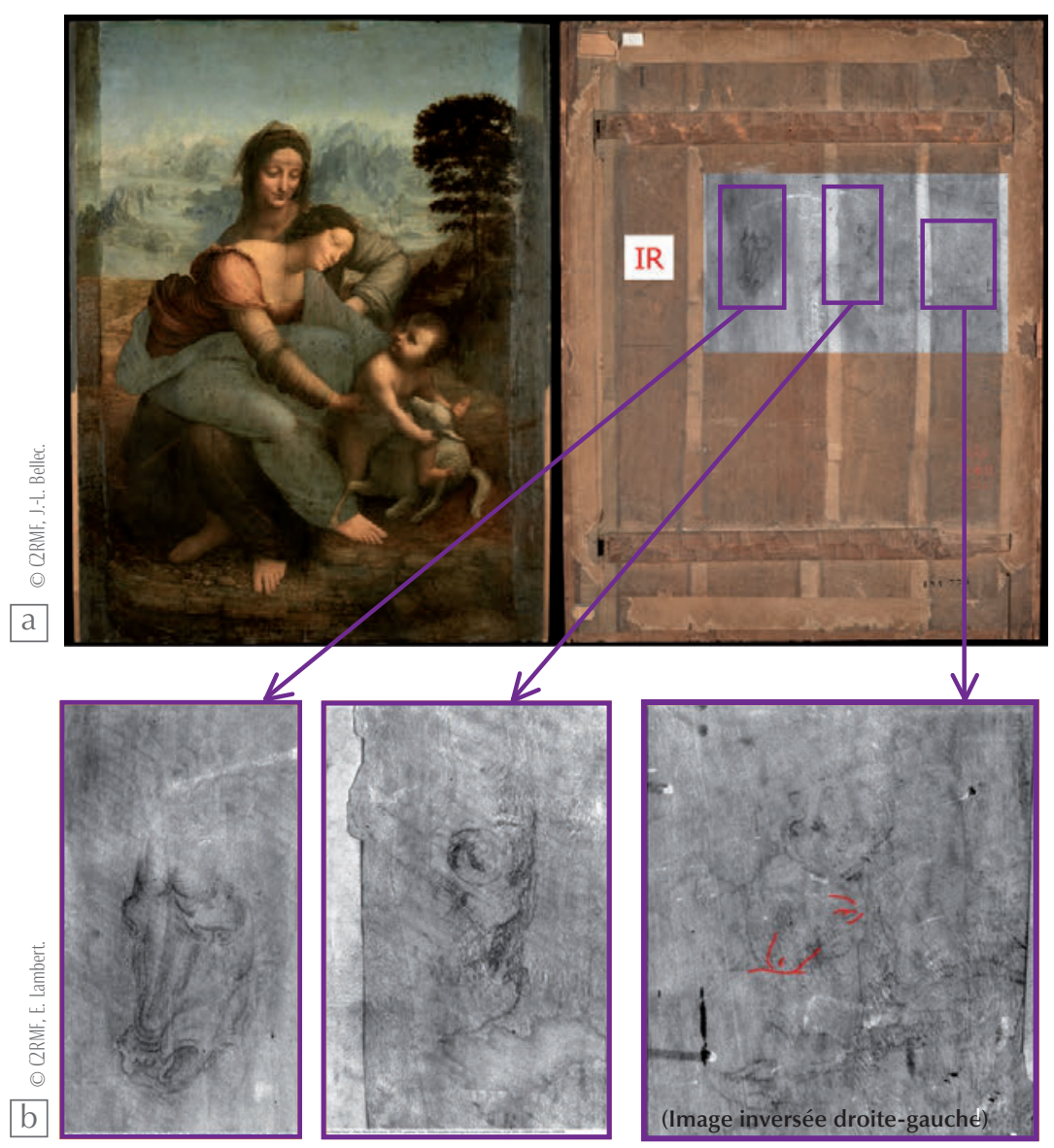

4. Photographies du tableau La Vierge, l'Enfant Jésus et Sainte Anne de Léonard de Vinci (hauteur 1684 mm, largeur 1263 mm). (a) Recto et verso en lumière visible avant restauration avec, en insert, le cliché en réflectographie infrarouge. (b) Détails du cliché en réflectographie infrarouge des dessins du verso. De gauche à droite : une tête de cheval, un demi-crâne, une esquisse de l'Enfant Jésus à l'agneau. La photo de droite a été inversée et la tête de l'agneau surlignée en rouge pour faciliter la lecture et la comparaison avec le recto de l'œuvre. informations sur le support (type de bois, tissage de la toile, réparations) et sur les différentes couches picturales (repentirs, changements dans la composition, réutilisation des supports) [4].

Les tubes de rayons $\mathrm{X}$ émettent un spectre quasi continu dont l'énergie maximum peut être ajustée entre 100 et $400 \mathrm{keV}$ suivant les besoins. Les objets massifs seront radiographiés avec des tubes de tension élevée et des temps de pose longs (parfois plusieurs heures), alors que pour les peintures on utilisera des tensions plus faibles et des temps plus courts (quelques minutes). Les rayonnements transmis sont enregistrés par des capteurs numériques ou sur des films. L'avantage de ces derniers est la très bonne résolution latérale, mais ils nécessitent en revanche du temps pour leur développement.

La transmission de rayons $\mathrm{X}$ suit la loi de Beer-Lambert : $\mathrm{N}=\mathrm{N}_{0} \exp (-\mu \mathrm{x})$, où $\mathrm{N}_{0}$ est le nombre de photons $\mathrm{X}$ incidents, $\mathrm{N}$ le nombre de photons $\mathrm{X}$ transmis, $\mu$ le coefficient d'absorption linéaire qui croît avec la densité électronique des atomes, et x l'épaisseur traversée. Ainsi, le nombre de photons qui vont impressionner le film dépend à la fois de la nature des matériaux au travers du coefficient d'absorption et de l'épaisseur des différentes parties de l'œuvre.

Pour une même épaisseur, on pourra discriminer des pigments à base de plomb ou de mercure comme le blanc de plomb $\left(2 \mathrm{PbCO}_{3} \cdot \mathrm{Pb}(\mathrm{OH})_{2}\right)$ ou le vermillon $(\mathrm{HgS})$, qui absorbent beaucoup plus les rayons $\mathrm{X}$ que les pigments composés d'atomes plus légers comme le blanc de zinc $(\mathrm{ZnO})$ ou le blanc de titane $\left(\mathrm{TiO}_{2}\right)$. En revanche, l'interprétation des clichés se complique quand de fortes épaisseurs compensent de faibles absorptions ou quand un matériau très absorbant recouvre l'ensemble de la peinture. Ce dernier cas est rencontré pour La Joconde, où une couche épaisse de blanc de plomb a été utilisée pour aplanir la planche servant de support (fig. 5). On distingue des plages blanches caractéristiques des couches épaisses de préparation qui comblent les irrégularités, mais qui masquent les contours du modèle. La radiographie met cependant en évidence le fil du bois, notamment les cernes du panneau de bouleau. Ce diagnostic de l'état de conservation permet de suivre une évolution éventuelle du support, en comparant la radiographie 
>>

avec les clichés réalisés lors d'examens précédents.

Pour le tableau Saint Jean-Baptiste, le résultat est similaire : les veines du bois, ici du noyer, ainsi que les trous laissés par les insectes xylophages sont perceptibles grâce à leur remplissage au blanc de plomb $^{(\mathrm{e})}$. On distingue aussi le monogramme CR (Collection Royale) peint au verso du tableau, mais qui n'est pas visible car recouvert d'un autre panneau de bois.

En ce qui concerne la peinture Dame priant, la radiographie contient de nombreuses informations. On peut y voir notamment un profond remaniement de l'œuvre. On découvre deux autres personnages en haut et à gauche de la Dame, ainsi qu'un fond uni sur la droite. Ces éléments indiquent une histoire mouvementée du tableau : Dame priant a été découpée dans une peinture de plus grand format, aujourd'hui disparue. Puis elle a été transformée en portrait, en plaçant le personnage au centre de la composition par l'adjonction d'une planche de bois au support original. Le fond a été ensuite recouvert d'une peinture foncée, et le visage ainsi que la collerette ont été rehaussés de blanc de plomb.

D'autres études radiographiques ont mis à jour de singulières pratiques, comme le réemploi de toiles déjà peintes. C'est le cas de Van Gogh qui réutilisait ses propres œuvres [5], ou de Picasso à qui il est arrivé d' "emprunter " des tableaux de la réserve du musée d'Antibes. Ainsi,
Le gobeur d'oursin, peint en 1946, recouvre le portrait du Général Vanderberg (d'auteur non connu), héros de la guerre de 1914, généreux donateur du musée [6].

\section{Des rayonnements pour l'analyse des peintures}

Les rayons $\mathrm{X}$ ne sont pas employés seulement pour la radiographie. Ils sont aussi utilisés comme sonde pour des techniques d'analyses élémentaire et structurale, comme la fluorescence $\mathrm{X}$ et la diffraction des rayons $X$. De récents développements ont permis de miniaturiser les appareils pour les déplacer au musée ou les emporter auprès des monuments ou sites naturels.

Ainsi, la Joconde et d'autres peintures de Léonard de Vinci ont pu être analysées in situ, au musée du Louvre, dans le cadre d'une thèse consacrée à la technique du sfumato, effet artistique vaporeux qui estompe les contours, adoucit les transitions et fond les ombres comme une fumée [7]. Les carnations (visages et mains) de sept tableaux ont été ainsi sondées par fluorescence $\mathrm{X}$ et donc sans prélèvement - exclus sur ces parties majeures de portrait -, pour déterminer la composition et l'épaisseur de chaque couche de matière [8]. Ces paramètres sont calculés à partir de l'intensité des rayons $\mathrm{X}$ émis par les éléments constituant les pigments et de leur absorption différentielle lors de la traversée des couches. Les résultats montrent que l'effet vaporeux est produit par la superposition de nombreuses microcouches de glacis, dont la couleur sombre est obtenue par l'ajout d'oxydes de fer et de manganèse.

Récemment, une nouvelle version du spectromètre de fluorescence $\mathrm{X}$ portable (voir la photo p. 101), permettant de balayer horizontalement et verticalement un faisceau collimaté de rayons $\mathrm{X}$, a donné lieu à la cartographie élémentaire de La Belle Ferronnière (L. de Vinci), avant sa restauration $[9,10,11]$. La répartition des éléments fer et cuivre montre que le fond sombre a été réalisé par la superposition de couches de peintures différentes: une première couche brune et homogène composée de terres $(\mathrm{Fe})$ et de noir a été recouverte d'une seconde couche à base de cuivre plus hétérogène, laissant apercevoir les coups de pinceau.

Les appareils portables sortent du laboratoire pour aller encore plus loin, par exemple pour accéder au patrimoine immobile comme les fresques des églises ou les représentations des grottes ornées. En Dordogne, un des berceaux de l'art pariétal en Europe, un appareil combinant fluorescence et diffraction des rayons $\mathrm{X}$ a été employé pour analyser les pigments noirs de la grotte de Rouffignac. Cette grotte recèle plus de 100 dessins, datés de 15000 ans environ, représentant majoritairement des mammouths (fig. 6). Par l'association des deux techniques, il a été possible d'accéder simultanément à la
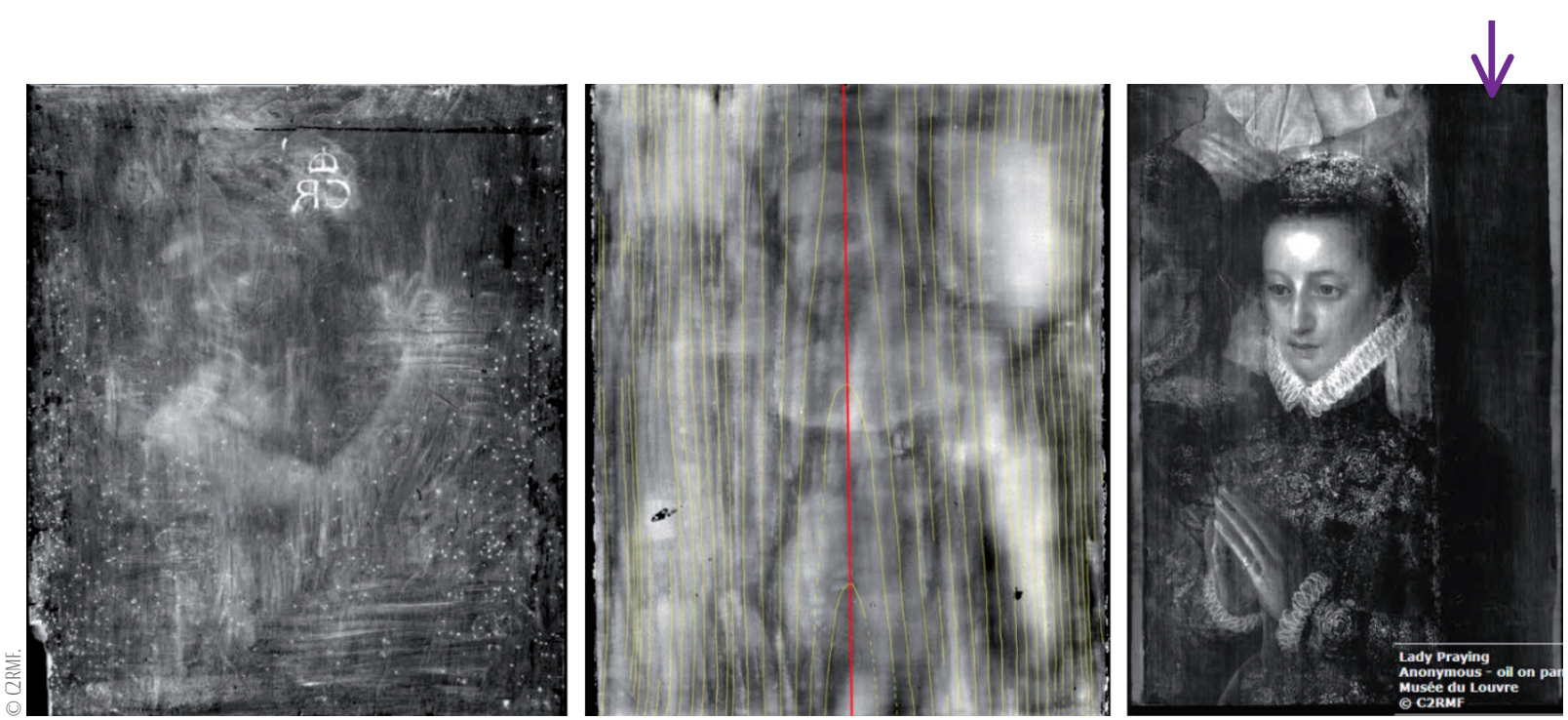

5. Radiographie X des trois tableaux présentés en figure 1. Sur le tableau de La Joconde (au centre), les traits jaunes indiquent les cernes du bois et le trait rouge l'axe central du panneau de peuplier. Pour Dame priant (à droite), la flèche pointe la planche de bois ajoutée au tableau initial. 


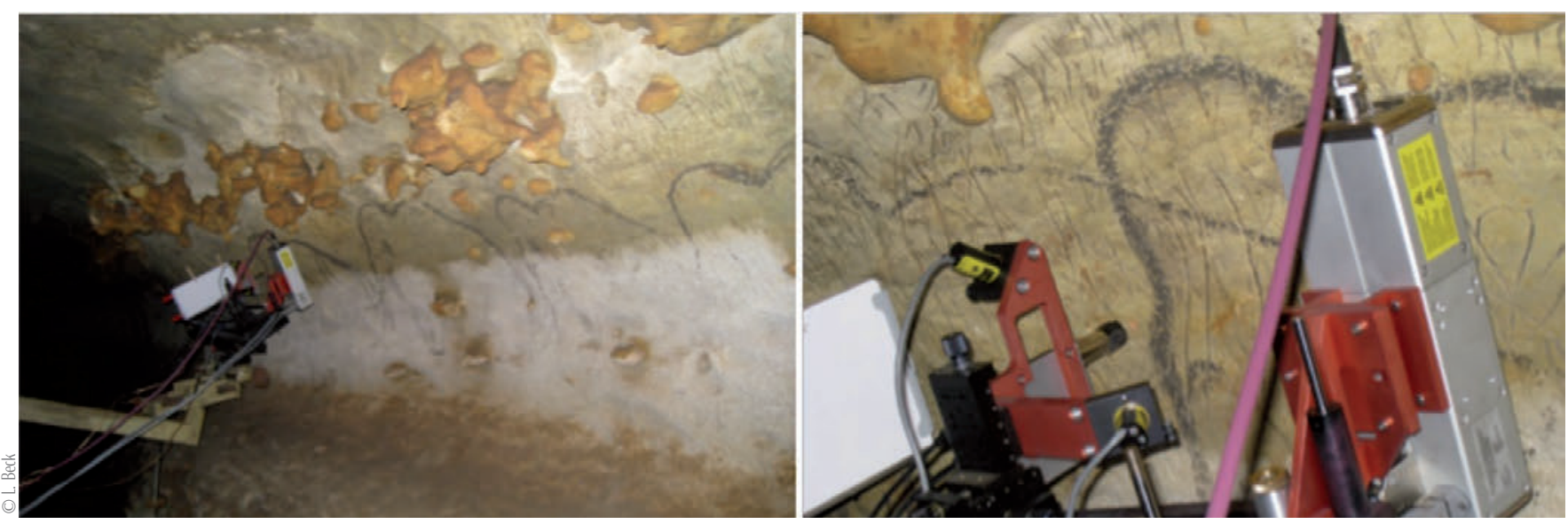

6. Analyse par fluorescence $X$ et diffraction des rayons $X$ des dessins préhistoriques de la grotte de Rouffignac (Dordogne, France) [12]. À gauche, l'appareil devant la frise des dix mammouths. À droite, détail montrant le tube à rayons $X$, le détecteur de fluorescence $X$ et le film pour l'enregistrement des anneaux de diffraction.

composition chimique et à l'identification des phases minéralogiques. Deux structures cristallographiques ont été déterminées pour les pigments : la pyrolusite (oxyde de manganèse) et la romanéchite (oxyde mixte de manganèse et de baryum). Ces deux composés, alliés au quartz, sont à la base de trois groupes de compositions distinctes, pouvant mettre en évidence des approvisionnements en matières premières ou des phases de réalisations différentes [12].

Dans le cas de La Frise des mammouths, une composition chimique relativement homogène (mélange pyrolusite-romanéchite, contenant environ $10 \%$ de $\mathrm{BaO}$ ) a été mesurée pour les dix dessins de la frise. La mise en évidence au sein de ce panneau d'une uniformité "chimique " est cohérente avec l'unité thématique et stylistique observée. En effet, tous les sujets sont dessinés à la même échelle et leur répartition est régulière et symétrique sur la paroi. Les analyses nous apprennent donc aussi que c'est vraisemblablement à partir d'un même bloc de pigment que les "crayons " ont été façonnés pour dessiner les dix mammouths. L'ensemble de ces résultats laisse imaginer une unité de conception et de réalisation.

Dans une autre partie de la grotte, le Grand Plafond, c'est un tout autre schéma qui se dessine : la diversité thématique des 65 dessins semble sans organisation apparente. Les premières analyses orientent vers deux groupes de composition, sans corrélation avec le type d'animal représenté. Les prochains résultats des mesures, toujours en cours, permettront peut-être de mieux comprendre les processus de mise en place des œuvres.

\section{Références}

1- Ouvrage collectif, Au cœur de la Joconde, Léonard de Vinci décodé, Gallimard, Paris (2006).

2• J.R.J. van Asperen de Boer, Applied Optics 7 (1968) 1711-1714.

3- Communiqué de presse du CNRS du 18/12/2008 www2.cnrs.fr/presse/communique/1486.htm

4 É. Martin et É. Ravaud, « La radiographie des peintures de chevalet », Techné $\mathbf{2}$ (1995) 158.

\section{5• J. Dick et al., Anal. Chem. 80 (2008) 6436-42.}

6• J.P. Mohen, Les sciences du patrimoine : identifier, conserver, restaurer, Odile Jacob (1999).

7• L. de Viquerie, « Propriétés physico-chimiques et caractérisation des matériaux du 'sfumato' ", thèse soutenue le lundi 2 novembre 2009. Université Pierre et Marie Curie, Paris 6.

8• L. de Viguerie et al., Angew. Chem. Int. Ed. 49 (2010) 6125-6128.

\section{Conclusion}

Les rayonnements visibles, infrarouges, ultraviolets et $\mathrm{X}$, apportent de précieuses informations sur les œuvres d'art et sur les objets archéologiques. Parmi les dizaines de milliers de clichés photographiques et radiographiques qui forment aujourd'hui le fond documentaire du C2RMF, les exemples présentés dans cet article n'offrent qu'un infime aperçu des potentialités de ces techniques [11].

C'est aussi sans compter sur la capacité des grands instruments qui, depuis deux décennies, apportent leur contribution au patrimoine culturel. Ainsi, en sondant la matière, les particules issues d'accélérateurs [13] et la lumière synchrotron [14] fournissent des outils complémentaires à l'imagerie et à l'instrumentation portable.
9• É. Ravaud et M. Eveno, “La Belle Ferronnière: a non invasive technical examination" in Leonardo da Vinci's technical practice - Paintings, drawings and influence, Michel Menu ed., Hermann, Paris, (2014), pp. 126-138.

10• « La Belle Ferronnière, études scientifiques et restauration ", Actualités (avril 2015), C2RMF. É. Ravaud et al., accepté pour publication dans Applied Physics A.

11• Des exemples complémentaires sont présentés dans I'article de L. de Viguerie, M. Alfeld et Ph. Walter dans ce numéro, p. 106.

12• L. Beck et al., Talanta 129 (2014) 459-464.

13• L. Beck, Nucl. Instr. Meth. Phys. Res. B 332 (2014) 439-444.

14• M. Cotte, Reflets de la physique, 34-35 (2013) 98-100. (a) Quelques définitions.

Peinture à l'huile : peinture constituée de couleurs broyées et agglutinées avec de l'huile siccative et véhiculées grâce à une essence volatile.

Glacis : couche de peinture à l'huile, légère et transparente. Il est utilisé pour obtenir profondeur et transparence.

Vernis : couche transparente de finition d'une peinture, qui protège la matière picturale du milieu ambiant. Il est obtenu en dissolvant une résine naturelle ou synthétique dans un diluant volatil.

(b) Un site interactif permet de visualiser quelques peintures en haute définition http://technologies.c2rmf.fr/showcase

(c) De rares pigments fluorescent sous UV comme, par exemple, le blanc de zinc $(\mathrm{ZnO})$.

(d) Repentir : modification d'une composition, lor de l'exécution d'un tableau, faite par l'auteur lui-même. Repeint : modification d'une composition, faite par une main différente de celle de l'auteur.

(e) Les détails des images peuvent être visionnés sur le site internet :

http://merovingio.c2rmf.cnrs.fr/iipimage/showcase/StJohnTheBaptist/explanation.html 